\title{
Convergence in the Linked Cluster Theorem for Many Body Fermion Systems
}

\author{
V. A. Malyshev \\ Department of Mathematics and Mechanics, Moscow State University, \\ SU-117234 Moscow, USSR
}

\begin{abstract}
We consider small coupling fermion systems with ultraviolet and space cutoffs and prove that they are asymptotically complete. The main ingredient is the convergence in the real time linked cluster theorem.
\end{abstract}

Here we prove some conjectures which are either explicit or implicit in the classical books by Friedrichs [1] and Hepp [2].

One of the main tools in the quantum many-body calculations is the linked cluster theorem (see Theorems 2.7 and 2.8 in [2]), which up to now was a purely formal result even with the adiabatic cutoff. Here the convergence of the corresponding series is proven. The corollary is asymptotic completeness. We stress that convergence is given in real time, using some experience in the euclidean region. In general, cluster expansions in real time (necessary for asymptotic completeness) seem to be more involved than in the euclidean region.

\section{Notations}

We use mostly notations and definitions of [2]. Let us consider the antisymmetric Fock space $\mathscr{F}=\mathscr{F}\left(L_{2}\left(R^{v}\right)\right)$ over $L_{2}\left(R^{v}\right)$; further on $v \geqq 3, a^{*}(f)$ and $a(f)$ being creation and annihilation operators, satisfying

$$
a^{*}(f) a(g)+a(g) a^{*}(f)=(f, g), \quad f, g \in L_{2}\left(R^{v}\right),
$$

where the scalar product $(f, g)$ in $L_{2}\left(R^{v}\right)$ is antilinear in the second argument. Let us denote by $\widetilde{\mathscr{F}}_{0}(S)$ the set of vectors

$$
\left(f_{0}, f_{1}, \ldots, f_{n}, 0,0, \ldots\right)
$$

in $\mathscr{F}$ such that $f_{n}\left(x_{1}, \ldots, x_{n}\right) \in S\left(R^{v n}\right)$.

We consider hamiltonains

$$
H=H_{0}+\lambda V
$$


on $\mathscr{F}$, where in the $k$-representation

$$
H_{0}=\int \omega(k) a^{*}(k) a(k) d k, \quad k \in R^{v}
$$

with the two cases: the massive case, with $\omega(k)=\left(m^{2}+k^{2}\right)^{1 / 2}$, and the massless case with $\omega(k)=k^{2}$,

$$
\begin{gathered}
V=\sum_{i=1}^{d} \int V_{i}\left(k_{1}, \ldots, k_{m_{\imath}}, k_{m_{i}+1}, \ldots, k_{m_{i}+l_{i}}\right) a^{*}\left(k_{1}\right) \ldots a^{*}\left(k_{m_{\imath}}\right) \\
\times a\left(k_{m_{i}+1}\right) \ldots a\left(k_{m_{i}+l_{i}}\right) d k_{1} \ldots d k_{m_{i}+l_{\imath}} \\
V_{i} \in S\left(R^{v\left(m_{i}+l_{\imath}\right)}\right) .
\end{gathered}
$$

It can be said that we consider interactions with an ultraviolet and space cutoff. We assume $V$ and hence $H$ to be selfadjoint. cutoff

Let us define, for $-\infty<s, t<\infty, \varepsilon \geqq 0$, an evolution operator without adiabatic

$$
U(t, s)=e^{i t H_{0}} e^{-i(t-s) H} e^{-i s H_{0}},
$$

and with adiabatic cutoff

$$
U^{(\varepsilon)}(t, s)=1-i \lambda \int_{s}^{t} d t^{\prime} V^{(\varepsilon)}\left(t^{\prime}\right) U^{(\varepsilon)}\left(t^{\prime}, s\right)
$$

It is well known that for finite $t$ and $s$ and $\varepsilon \geqq 0$,

$$
U^{(\varepsilon)}(t, s)=1+\sum_{n=1}^{\infty}(-i \lambda)^{n} \int_{s}^{t} d t_{1} \ldots \int_{s}^{t_{n}-1} d t_{n} V^{(\varepsilon)}\left(t_{1}\right) \ldots V^{(\varepsilon)}\left(t_{n}\right)
$$

and the series is norm convergent.

The integrand in (3) is the product of Wick monomials and can be represented as the sum

$$
V^{(\varepsilon)}\left(t_{1}\right) \ldots V^{(\varepsilon)}\left(t_{n}\right)=\sum_{G} W_{G}\left(t_{1}, \ldots, t_{n}\right)
$$

of the Wick monomials $W_{G}$ which are labeled by Friedrichs diagrams $G$ (see [2]). So

$$
\begin{aligned}
W_{G}= & (-1)^{\pi(G)} \prod_{v=1}^{n} V_{i(v)}^{(\varepsilon)}\left(k_{v}, 1, \ldots, k_{\left.v, m_{\imath(v)}+l_{\imath(v)}\right)}\right. \\
& \times \prod_{\text {int }} \delta\left(k_{v p}-k_{v^{\prime} q}\right) e^{i\left(t_{v^{\prime}}-t_{v}\right) \omega\left(k_{v p}\right)} d k_{v p} d k_{v^{\prime} q} \\
& \times \prod_{\mathrm{ext}} a^{\#}\left(k_{v p}\right) e^{ \pm t_{v} i \omega\left(k_{v p}\right)} d k_{v p},
\end{aligned}
$$

where the first product is over all vertices of the diagram $G$ and we call $k_{v i}$ the variable of the $i^{\text {th }}$ leg in the vertex $v$. The second product is over all internal lines $\left(v p, v^{\prime} q\right)$ of $G$ contracting left (creation) legs $v p$ and right (annihilation) legs $\left(v^{\prime} q\right)$. The third product is over all external (noncontracted) lines (legs) and \pm depends on whether $\#=*$ or not.

Let us denote

$\left(V\left(t_{1}\right) \ldots V\left(t_{n}\right)\right)_{c}$ - the sum over all connected diagrams in (4);

$(\ldots)_{00}$ - the sum over all connected diagrams without external lines in (4);

$(\ldots)_{L}=(\ldots)_{c}-(\ldots)_{00}$ - over all connected diagrams with at least one external line;

$(\ldots)_{C R}$ - over all diagrams of class $(\ldots)_{L}$ which external lines are creation lines. 


\section{Results}

Theorem 1. There exists $\lambda_{0}>0$ such that for $|\lambda|<\lambda_{0}$ and either $-\infty<s, t<\infty$ and $\varepsilon \geqq 0$ or $-\infty \leqq s, t \leqq \infty$ and $\varepsilon>0$ the series

$$
\sum_{n=1}^{\infty}(-i \lambda)^{n} \int_{s}^{t} d t_{1} \ldots \int_{s}^{t_{n}-1} d t_{n}\left(V^{(\varepsilon)}\left(t_{1}\right) \ldots V^{(\varepsilon)}\left(t_{n}\right)\right)_{c} \stackrel{\text { def }}{=} U^{(\varepsilon)}(t, s)_{c}
$$

is norm convergent, $\lambda_{0}$ does not depend on $s, t, \varepsilon$. The same is true if instead $(\ldots)_{c}$ one takes $(\ldots)_{00},(\ldots)_{L},(\ldots)_{C R}$.

We suppose further on $V$ to be even (i.e. $m_{i}+l_{i}$ are even for all $i$ ). Using Theorem 1 one can prove nonformally

Theorem 2 (Linked cluster theorem, see Theorem 2.7 in [2]). Under the conditions of Theorem 1,

$$
U^{(\varepsilon)}(t, s)=: \exp U^{(\varepsilon)}(t, s)_{c}:
$$

where the series in the right-hand side is norm convergent.

Let us denote

$$
T_{t s}^{(\varepsilon)}=\frac{U^{(\varepsilon)}(t, s)}{\left(\Omega, U^{(\varepsilon)}(t, s) \Omega\right)},
$$

where $\Omega$ is the vacuum in $\mathscr{F}$.

Theorem 3. If $v \geqq 3$, then for both the massive and massless case there exists $\lambda_{0}>0$ such that for $|\lambda|<\lambda_{0}$ there exist

$$
\begin{aligned}
& s-\lim _{\varepsilon \rightarrow 0} T_{0, \pm \infty}^{(\varepsilon)} \stackrel{\text { def }}{=} T^{ \pm} \quad \text { (direct morphisms), } \\
& s-\lim T_{\varepsilon \rightarrow 0} T_{ \pm \infty, 0}^{(\varepsilon)} \stackrel{\text { def }}{=} \widehat{T}^{ \pm} \quad \text { (inverse morphisms). }
\end{aligned}
$$

Theorem 4. Under the conditions of Theorem 3 wave function renormalisation

$$
Z^{-1}=\left\|\exp \sum_{n=1}^{\infty}(-\lambda)^{n}(\Gamma(V \ldots \Gamma(V)))_{C R} \Omega\right\|^{2},
$$

where $n$ is the number of Friedrich's operations $\Gamma$, is finite and $\sqrt{Z} T^{ \pm}$are unitary and provide unitary equivalence

$$
H T^{ \pm}=T^{ \pm}\left(H_{0}+\varepsilon_{\lambda}\right),
$$

where $\varepsilon_{\lambda}$ is given by the convergent Goldstone series

$$
\varepsilon_{\lambda}=\left(\Omega, \lambda V \perp T^{ \pm} \Omega\right) .
$$

( $V \perp T$ is the left connected product, see $[1,2]$.)

Remark 1. Unitary equivalence of $H$ and $H_{0}+\varepsilon_{\lambda}$ was known for the massive case $($ see $[3,4])$ and for the massless case without vacuum polarisation [3]. 


\section{Proof of Theorem 1: "Time-Mode Clusters"}

The only difficulty is the number of the diagrams. We circumvent this difficulty by making fermionic cancellations. These cancellations are made in the "time-mode" clusters.

For the sake of concreteness we consider the case $\varepsilon=0,-\infty<s, t<\infty, v \geqq 3$. In this case we shall prove

Lemma 1. The norm of the $n^{\text {th }}$ term of (6) has the bound $|s-t| C^{n} \lambda^{n}$, where $C$ does not depend on $s, t, n$.

Time clusters are specified by the notion of the sector.

Partitions. Indices $I, \ldots, n$ are the vertices of the diagrams. Any subset $\alpha=\left(\alpha_{1}, \ldots, \alpha_{k}\right), \alpha_{1}<\ldots<\alpha_{k}$, of $(1, \ldots, n)$ defines the partition of $(1, \ldots, n)$ onto the intervals

$$
\begin{aligned}
\Delta_{1} & =\left[1, \alpha_{1}\right)=\left\{i: 1 \leqq i<\alpha_{1}\right\}, \ldots \\
\Delta_{k} & =\left[\alpha_{k-1}, \alpha_{k}\right), \Delta_{k+1}=\left[\alpha_{k}, n\right] .
\end{aligned}
$$

Sectors. Any $\alpha$ defines the subset $\mathfrak{A}_{\alpha}$ of

$$
\mathfrak{U}_{s t}^{n}=\left\{\left(t_{1}, \ldots, t_{n}\right): s<t_{1}<\ldots<t_{n}<t\right\}
$$

which we call a sector and which is uniquely defined by the following properties:

1. If $i, j$ belong to the same interval $\Delta_{l}$ of the partition $\alpha$, then there exists an integer $M$ such that

$$
t_{i}, t_{j} \in(M, M+1) \stackrel{\text { def }}{=} \widehat{\Delta}_{l} .
$$

2) If $i, j$ belong to different $\Delta_{m}$ and $\Delta_{l}$ respectively, then $t_{i}, t_{j}$ belong to different $(M, M+1)=\widehat{\Delta}_{m},(L, L+1)=\widehat{\Delta}_{l}$, i.e. $M \neq L$.

It is clear that $\bigcup_{\alpha} \mathfrak{A}_{\alpha}=\mathfrak{M}_{t s}^{n}$. Let us call $\Delta_{l}$ the $l^{\text {th }}$ group of the sector $\mathfrak{A}_{\alpha}$.

Subsectors. A subsector $\mathfrak{A}_{\alpha}\left(M_{1}, \ldots, M_{k+1}\right)$ is defined by $\alpha$ and by integers $M_{1}>M_{2}>\ldots>M_{k+1}$. So this subsector is the set of all $t_{1}>\ldots>t_{n}$ such that if $i \in \Delta_{j}$, then $t_{i} \in\left(M_{j}, M_{j+1}\right)$.

Modes. Let $N=\left(N^{1}, \ldots, N^{v}\right) \in Z^{v}, k=\left(k^{1}, \ldots, k^{v}\right) \in R^{v} . N$-mode is the function on $R$

$$
e_{N}(k)=\prod_{j=1}^{v} \chi_{B}\left(k^{j}\right) \exp \frac{2 \pi i N^{j} k^{j}}{A},
$$

where $\chi(k)$ is $C_{0}^{\infty}$-function, $0 \leqq \chi(k) \leqq 1, \chi(k)=1$ for $|k| \leqq B$ and 0 for $|k| \geqq B+1$. We choose $B$ so that for any $i$

$$
\operatorname{supp} V_{i} C(-B, B)^{v\left(l_{i}+m_{i}\right)},
$$

and choose $A>B$, for simplicity we assume $V_{i} \in C_{0}^{\infty}\left(R^{v\left(l_{i}+m_{i}\right)}\right)$.

Let us fix subsector $\mathfrak{H}_{\alpha}\left(M_{1}, \ldots, M_{k+1}\right)$. Any $t_{v} \in\left(M_{l}, M_{l+1}\right)$ can be represented as

$$
t_{v}=M_{1}+\Delta t_{v}, \quad \Delta t_{v}=M_{l}-t_{v} .
$$


So for any leg $p$ of the vertex $v$ we can write

$$
\exp \left(i t_{v} \omega\left(k_{v p}\right)\right)=\exp \left(i M_{l} \omega\left(k_{v p}\right)\right) \exp \left(i \Delta t_{v} \omega\left(k_{v p}\right)\right) .
$$

We attribute the second factor in the right-hand side of (11) to $V_{i(v)}$ in (5) and next we come to

Expansion in Modes. For any $v$ we use the expansion

$$
V_{i(v)}\left(k_{v 1}, \ldots, k_{v, m_{i(v)}+l_{i}(v)}\right) \prod_{p} \exp \left(i \Delta t_{v} \omega\left(k_{v p}\right)\right)=\prod_{p}\left(\sum_{N \in Z^{v}} c_{c p, N} e_{N}\left(k_{v p}\right)\right) .
$$

Let us note that for any $\gamma>0$ there exists a constant $C(\gamma)$ such that uniformly in $v, p$, $\left|\Delta t_{v}\right| \leqq 1$, constants $c_{v p, N}\left(\Delta t_{v}\right)$ satisfy the bound

$$
\left|c_{v p, N}\right| \leqq \frac{C(\gamma)}{|N|^{\gamma}}, \quad|N|=\sum_{i=1}^{v} N^{i}
$$

This is proved by integration by parts. Further on we fix $\gamma>v+2$.

Expansion in "Time-Mode" Clusters. Using the preceding definitions we can represent $n^{\text {th }}$ term of the series (6) as follows

i.e.

$$
\sum_{\mathfrak{A}_{\alpha} M_{1}, \ldots, M_{k+1}} \sum_{\text {modes }} \int_{\left|\Delta t_{v}\right| \leqq 1} \prod d\left(\Delta t_{v}\right) \sum_{G} W_{G},
$$

1. sum over sectors;

2. sum over subsectors of the given sector, i.e. sum over $M_{1}, \ldots, M_{k+1}$,

3. sum over modes, i.e. over $N_{v p}$,

4. integration inside subsector,

5. sum over admissible diagrams, where

$$
\begin{aligned}
W_{G}= & \prod_{\text {int }} \delta\left(k_{v p}-k_{v^{\prime} q}\right) \exp \left(i\left(M_{l}-M_{l^{\prime}}\right) \omega\left(k_{v p}\right)\right) c_{v p, N_{v p}} \\
& \times c_{v^{\prime} q, N_{v^{\prime} q}} e_{N_{v p}}\left(k_{v p}\right) e_{N_{v^{\prime} q}}\left(k_{v^{\prime} q}\right) d k_{v p} d k_{v^{\prime} q} \prod_{\mathrm{ext}} a^{\#}\left(k_{v p}\right) \\
& \times \exp \left( \pm i t_{v} \omega\left(k_{v p}\right)\right) c_{v p, N_{v p}} e_{N_{v p}}\left(k_{v p}\right) d k_{v p}(-1)^{\pi(G)} .
\end{aligned}
$$

We shall obtain now cancellations of some diagrams. Let us fix for any group $\Delta_{l}$ subsets $A_{l}$ and $B_{l}$ of left and correspondingly right legs of vertices of this group. Let for the given sector $\alpha \mathfrak{B}\left(\alpha,\left\{A_{l}\right\},\left\{B_{l}\right\}\right)$ be the set of all admissible diagrams $G$ such that exactly creation legs from $\bigcup A_{l}$ are contracted with some annihilation legs (i.e. creation legs not belonging to $\bigcup A_{l}$ are not contracted) and similarly for $\bigcup B_{l}$.

Lemma 2. Let the sector, subsector and the set $\left\{N_{v p}\right\}$ of modes be fixed. Then if for some $l$ there are either at least two equal modes in $A_{l}$, i.e. if $N_{v p}=N_{v^{\prime} q}$ for some vp, $v^{\prime} q \in A_{l}$, or at least two equal modes in $B_{l}$ then

$$
\sum_{G \in \mathfrak{B}\left(\alpha,\left\{A_{1}\right\},\left\{B_{1}\right\}\right)} W_{G}=0 .
$$

The proof is evident: let us consider two legs in e.g. $A_{l}$ with equal modes and two diagrams in which these two modes are contracted with two other legs in two different ways (all other contractions are the same). Then these two diagrams exactly cancel. 
So further on we can assume that inside any $A_{l}$ and inside any $B_{l}$ all modes are different and we use the bound

$$
\prod\left|c_{v, p, N_{v p}}\right| \leqq\left[\prod \frac{C(\gamma)}{\left|N_{v p}\right|^{\gamma-1}}\right] \frac{1}{A_{l} ! B_{l} !},
$$

where both products are over $(v, p) \in A_{l} \cup B_{l}$.

Having made these cancellations we shall use the bound for (14)

$$
\sum_{\mathfrak{M}_{\alpha} M_{1}, \ldots, M_{k+1}} \sum_{\text {modes }} \int_{\left|\Delta t_{v}\right| \leqq 1} \prod d\left(\Delta t_{v}\right) \sum_{G}\left\|W_{G}\right\| .
$$

Lemma 3. [5]

$$
\left|\int_{R^{\nu}} d k e_{N}(k) e_{M}(k) e^{i t \omega(k)}\right| \leqq \frac{C}{(1+|t|)^{v / 2}},
$$

where $C$ does not depend on $N$ and $M$.

This gives

$$
\left\|W_{G}\right\| \leqq \prod_{\text {int }} \frac{C}{\left|M_{l(v, p)}-M_{l\left(v^{\prime}, q\right)}\right|^{v / 2}},
$$

where $l(v, p)$ means that leg $(v, p)$ belongs to the group $\Delta_{l}$. Sum over sectors and integration in (17) give at most $2^{n}$ and we shall obtain the bound of the following expression uniform in $\alpha$ :

$$
\sum_{\left\{A_{l}, B_{l}\right\}} \frac{1}{\left|A_{l}\right| !\left|B_{l}\right| !} \sum_{\left\{N_{v p}\right\}}\left[\prod \frac{C(\gamma)}{\left|N_{v p}\right|^{\gamma-1}}\right]_{M_{1}, \ldots, M_{k+1}} \sum_{G} \prod_{\text {int }} \frac{C}{\left|M_{l(v, p)}-M_{l\left(v^{\prime}, q\right)}\right|^{v / 2}} .
$$

Again the second sum and $\sum_{\left\{A_{l}, B_{l}\right\}} 1$ give $C^{n}$. Let us consider the factor-graph

$$
\Gamma=G /\left\{\Delta_{1}, \ldots, \Delta_{k+1}\right\}
$$

with $k+1$ vertices, i.e. we identify vertices of $\Delta_{l}$ for any $l$. Then the number of graphs $G$ with the given factor-graph $\Gamma$ does not exceed $C^{n} \prod\left|A_{l}\right| !\left|B_{l}\right|$ !

So we are left with the sum over $M_{1}, \ldots, M_{k+1}$ and over factorgraphs $\Gamma$.

Lemma 4. Let us fix $M_{1}$. Then

$$
\sum_{\Gamma} \sum_{M_{2}, \ldots, M_{k+1}} \frac{1}{\left|M_{l(v, p)}-M_{l\left(v^{\prime}, q\right)}\right|^{v / 2}} \leqq\left(\sum_{M=-\infty}^{\infty} \frac{C}{(1+|M|)^{v / 2}}\right)^{r n}
$$

where $r=\max m_{i}=\max l_{i}$.

This is the standard result in cluster expansion techniques [6]. This completes the proof of Theorem 1, as summation over $M_{1}$ gives the factor $|s-t|$.

\section{Asymptotic Completeness}

The formal proof of Theorem 2 is in [2]. As $U(t, s)_{c}$ is the norm convergent series in annihilation and creation operators then so is $\exp U(t, s)_{c}$. Wick ordering does not change the upper bound of the norm and so Theorem 2 follows from Theorem 1. 
Remark 2. If $v=1,2$ then Theorems 1 and 2 are also true but with a worse bound than in Lemma 1. The proof repeats Sect. 3.

Proof of Theorem 3. We claim that

$$
\underset{\varepsilon \rightarrow 0}{\operatorname{norm}-\lim } \sum_{n=1}^{\infty}(-i \lambda)^{n} \int_{ \pm \infty}^{0} d t_{1} \ldots \int_{ \pm \infty}^{t_{n-1}} d t_{n}\left(V^{(\varepsilon)}\left(t_{1}\right) \ldots V^{(\varepsilon)}\left(t_{n}\right)\right)_{L} \psi
$$

exists where

$$
\psi=a^{*}\left(g_{1}\right) \ldots a^{*}\left(g_{r}\right) \Omega \in \mathscr{F}_{0}(S) .
$$

Let us consider the $n^{\text {th }}$ term of (19). First we restrict ourselves to the class of diagrams with the external annihilation line

$$
a\left(k_{v p}\right) \exp \left(-i t_{v} \omega\left(k_{v p}\right)-\varepsilon\left|t_{v}\right|\right)
$$

with fixed $v, p$. We repeat the proof of Theorem 1 with the extra factor $\left|M_{v}\right|^{-v / 2}$, which we get from contracting $a\left(k_{v p}\right)$ with some $a^{*}\left(g_{i}\right)$. With this extra factor we get the convergence uniform in $0 \leqq s \leqq \infty$ for $t=0$.

Next let us consider diagrams without annihilation external lines and having some creation external lines

$$
\prod_{v, p} a^{*}\left(k_{v p}\right) e^{i t_{v} \omega\left(k_{v p}\right)-\varepsilon\left|t_{v}\right|}
$$

We represent

$$
e^{i t_{v} \omega-\varepsilon\left|t_{v}\right|}=\exp \left(i t_{1} \omega-\varepsilon\left|t_{1}\right|+\sum_{j=2}^{v}(i \omega-\varepsilon)\left(t_{j}-t_{j-1}\right)\right)
$$

and integrate out $t_{1}$,

$$
\lim _{\varepsilon \rightarrow 0} i \int_{ \pm \infty}^{0} d t_{1} \exp \left(i t_{1}\right) \sum_{v, p} \omega\left(k_{v p}\right)-\varepsilon\left|t_{1}\right|=\frac{1}{\sum_{v, p} \omega\left(k_{v p}\right)} .
$$

In the massive case (20) belongs locally to $L_{2}$ and the remaining part of the proof repeats Sect. 3 .

Trouble in (20) can appear only if $\sum_{v, p}$ contains exactly one summand. But this is possible only if the degree of some $V_{i}$ is not even.

Under the same reasons there exist

$$
T^{ \pm} \psi=\underset{\varepsilon \rightarrow 0}{\text { norm-line }}: \exp U^{(\varepsilon)}(0, \pm \infty)_{L}:
$$

It is easy to see from the proof that quite similarly one can prove existence of $\widehat{T}^{ \pm} \psi$, $\hat{T}^{ \pm} T^{ \pm} \psi, T^{ \pm} \hat{T}^{ \pm} \psi$ on $\mathscr{F}_{0}(S)$ and also the fact that $Z^{-1}$ is finite. From the known identities for $U^{(\varepsilon)}(t, s)$ for $\varepsilon>0$ (in particular unitarity, see [2]) it follows then that $\sqrt{Z} T^{ \pm}$conserve the norm on $\mathscr{F}_{0}(S)$, hence are unitary.

The formal identities which prove Theorem 4 are well known (see [2]). The fact that they are convergent can be easily checked from the above considerations.

Remark 3. For $v=1,2$ we cannot prove convergence of the series even for direct morphisms, but the existence of direct morphisms can be easily proved by Cook's method. 
Remark 4. The class of functions $\omega(k)$ for which Theorems 1-4 take place can be described by two conditions: stationary phase estimate in Lemma 3 and local square integrability of (20).

\section{References}

1. Friedrichs, K.: Perturbation of spectra in Hilbert space. Providence: Am. Math. Soc. 1965

2. Hepp, K.: Théorie de la renormalisation. Berlin, Heidelberg, New York: Springer 1969

3. Botvich, D.D., Malyshev, V.A.: Commun. Math. Phys. 91, 301-312 (1983)

4. Aizenstadt, V.V.: Usp. Math. Nauk (to appear)

5. Reed, M., Simon, B.: Methods of modern mathematical physics, Vol. 3. New York: Academic Press 1978

6. Malyshev, V.A., Minlos, R.A.: Gibbs random fields. The method of cluster expansions. Dordrecht: Reidel (to appear)

Communicated by J. Fröhlich

Received February 29, 1988 